\title{
Mirror skin effect and its electric circuit simulation
}

\author{
Tsuneya Yoshida ${ }^{\circ}$, Tomonari Mizoguchi ${ }^{\circ}$, and Yasuhiro Hatsugai $\odot$ \\ Department of Physics, University of Tsukuba, Ibaraki 305-8571, Japan
}

(Received 27 December 2019; accepted 29 April 2020; published 17 June 2020)

\begin{abstract}
We analyze the impacts of crystalline symmetry on non-Hermitian skin effects. Focusing on mirror symmetry, we propose another type of skin effect, a mirror skin effect, which results in a significant dependence of the energy spectrum on the boundary condition only for the mirror invariant line in the two-dimensional Brillouin zone. This effect arises from the topological properties characterized by a mirror winding number. We further reveal that the mirror skin effect can be observed for an electric circuit composed of negative impedance converters with a current inversion where switching the boundary condition significantly changes the admittance eigenvalues only along the mirror invariant lines. Furthermore, we demonstrate that extensive localization of the eigenstates for each mirror sector results in an anomalous voltage response.
\end{abstract}

DOI: 10.1103/PhysRevResearch.2.022062

Introduction. The topological properties [1-3] of systems have become a central issue in condensed matter systems because of their remarkable ubiquity. Topological phenomena can be observed even for classical systems (e.g., photonic systems [4-6], mechanical systems [7-11], electric circuits $[12,13]$, etc.), which are mathematically described by an eigenvalue problem. Among the extensive studies of topological physics, one significant progress is the discovery of topological crystalline insulators [14-17], which has elucidated that topological properties can be enriched by crystalline symmetry [18-27]. A prime example of a topological crystalline insulator is SnTe [16,17] where the mirror Chern number topologically protects two surface Dirac cones.

Along with the above significant progress, non-Hermitian topological systems have been extensively studied, and a variety of novel phenomena have been discovered [28-45]. An important difference in non-Hermitian topological systems is that there exist two types of gaps [46], a line gap $[47,48]$ and a point gap $[49,50]$. The line-gap topology indicates the presence of a Hermitian counterpart. The pointgap topology protects non-Hermitian band touching in the bulk [51-59], such as exceptional points, etc. Other unique phenomena induced by non-Hermiticity, on which we focus in this Rapid Communication, can be observed for a system with boundaries [60-68]. In particular, it has been elucidated that the non-Hermitian skin effect is induced by nontrivial point-gap topology; the winding number characterizes the non-Hermitian skin effect of class A (no symmetry) [69-71]. The mathematically rigorous proof of the above relation has been obtained in Ref. [71] for class A. In addition, a $\mathbb{Z}_{2}$ skin effect with time-reversal symmetry has been proposed [71].

Published by the American Physical Society under the terms of the Creative Commons Attribution 4.0 International license. Further distribution of this work must maintain attribution to the author(s) and the published article's title, journal citation, and DOI.
The above progress for Hermitian and non-Hermitian systems lead us to the following issue: understanding the impacts of crystalline symmetry on non-Hermitian topological properties. This issue is crucial because a variety of novel topological phenomena are expected as are the cases for Hermitian systems. In spite of its significance, the above issue has not been sufficiently explored. In particular, there are few works elucidating novel skin effects induced by crystalline topology in the bulk.

Therefore, in this Rapid Communication, we analyze the effects of mirror symmetry on non-Hermitian skin effects, shedding light on the interplay between crystalline symmetry and non-Hermitian topology. Our analysis discovers another type of skin effect, a mirror skin effect, which results in a significant dependence of the energy spectrum on the boundary condition only along mirror invariant lines in the twodimensional Brillouin zone. Here, by the energy spectrum, we denote the spectrum of the non-Hermitian Hamiltonian. We also elucidate that a mirror winding number characterizes this skin effect. We verify the mirror skin effect by numerically diagonalizing a tight-binding model with the mirror winding number taking one. We note that the mirror skin effect cannot be observed in the absence of mirror symmetry, meaning that mirror symmetry is essential. Furthermore, by making use of the ubiquity of the topological phenomenon, we theoretically suggest that the mirror skin effect can be observed for an electric circuit composed of negative impedance converters with current inversion (see Fig. 1). In this system, switching the boundary conditions drastically changes the impedance for the mirror invariant lines, which serves as distinct evidence of the mirror skin effect for an electric circuit.

Theory of mirror skin effect. Let us first elucidate that the topology protected by mirror symmetry induces a skin effect.

For comparison, we start with a brief review of the ordinary skin effect for symmetry class A. Consider a two-dimensional system under a periodic boundary condition for the $x$ direction which can be regarded as a set of one-dimensional systems aligned along the $x$ direction in the momentum space. When the winding number $v_{\text {tot }}\left(k_{x}\right)$ takes a finite value for the sub- 


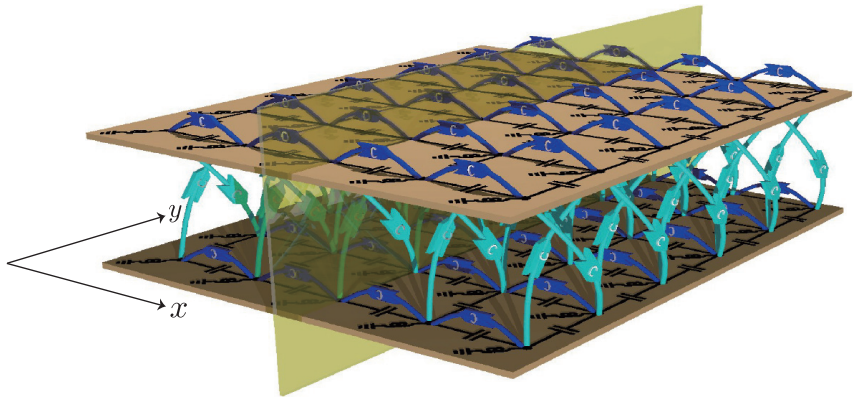

FIG. 1. Sketch of the electric circuit showing the mirror skin effect. The blue (cyan) symbols represent negative impedance converters with current inversion connecting intralayer (interlayer) nodes, respectively. We refer to the upper (lower) layer as A (B), respectively. The black symbols denote capacitors. Dots represent nodes. This system preserves the mirror symmetry whose mirror plane is illustrated with a vertical yellow plane.

system with a given momentum $k_{x}$, the skin effect occurs; the energy spectrum significantly changes by switching the boundary condition for the $y$ direction [i.e., the periodic boundary condition (PBC) to the open boundary condition $(\mathrm{OBC})]$. This relation can be understood with a topological deformation; each subsystem for a given momentum $k_{x}$ is topologically deformed into the Hatano-Nelson model [or its variant showing a higher value of the winding number $\left.v_{\text {tot }}\left(k_{x}\right)\right]$ exhibiting the skin effect. The above fact indicates that the ordinary skin effect of class $\mathrm{A}$ is induced by the winding number $v_{\text {tot }}\left(k_{x}\right)$. (For later use, we call it the total winding number.)

Now, we elucidate a skin effect whose topological properties are protected by mirror symmetry; in contrast to the ordinary skin effect mentioned above, the mirror skin effect elucidated below occurs even when the total winding number is zero for arbitrary momenta $k_{x}$. We call this skin effect the mirror skin effect. In the rest of this Rapid Communication, we assume $v_{\text {tot }}\left(k_{x}\right)=0$ unless otherwise stated.

First, we note that the presence of mirror symmetry results in an additional topological invariant. Consider the Hamiltonian which is invariant under applying the mirror operator $M_{x}$,

$$
\begin{aligned}
M_{x} H(\boldsymbol{k}) M_{x}^{-1} & =H\left(M_{x} \boldsymbol{k}\right), \\
M_{x} & =U_{m} P_{x},
\end{aligned}
$$

where $P_{x}$ flips the momentum $\boldsymbol{k}:=\left(k_{x}, k_{y}\right) \rightarrow M_{x} \boldsymbol{k}:=$ $\left(-k_{x}, k_{y}\right) . U_{m}$ is an unitary matrix satisfying $U_{m}^{2}=1$. Along the mirror invariant line specified by $k_{x}^{*}$, the Hamiltonian can be block-diagonalized for the plus and the minus sectors of the operator $M_{x}$. Thus, besides the total winding number $v_{\text {tot }}$, the following mirror winding number can be defined,

$$
v_{M}=\left(v_{+}-v_{-}\right) / 2 .
$$

Here, $v_{ \pm}\left(k_{x}^{*}\right)$ denotes the winding number computed with the block-diagonalized Hamiltonian $H_{ \pm}\left(k_{x}^{*}, k_{y}\right)$ for each sector,

$$
\nu_{ \pm}\left(k_{x}^{*}\right)=\int \frac{d k_{y}}{2 \pi i} \partial_{k_{y}} \log \operatorname{det}\left[H_{ \pm}\left(k_{x}^{*}, k_{y}\right)-E_{\mathrm{pg}}\right],
$$

where $E_{\mathrm{pg}}$ is the reference energy for the point gap [72,73]. We note that the total winding number is computed with $v_{\text {tot }}\left(k_{x}^{*}\right)=v_{+}\left(k_{x}^{*}\right)+v_{-}\left(k_{x}^{*}\right)$ for the mirror invariant lines. For
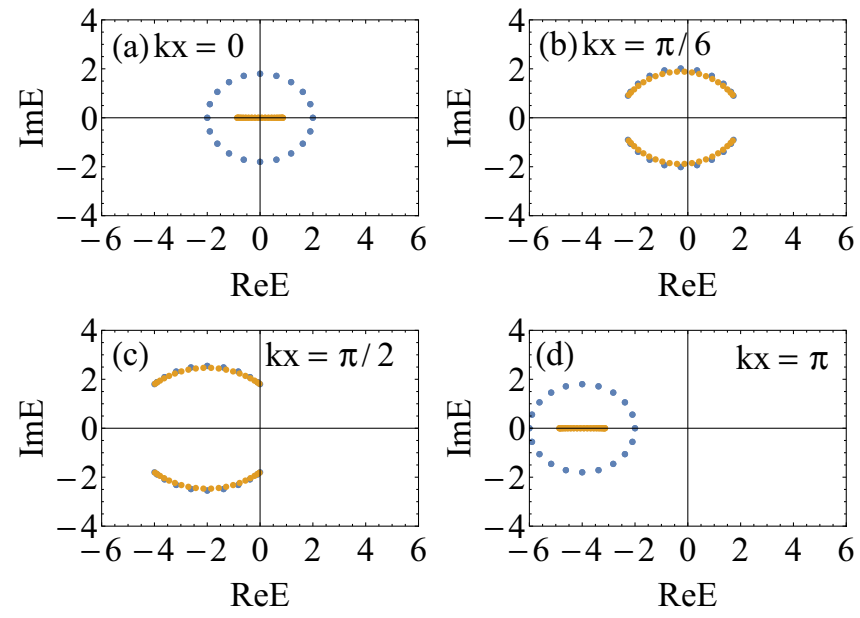

FIG. 2. Energy spectrum of the Hamiltonian (4) for $(t, \mu, \Delta)=$ $(1,2,1.8)$. Blue (orange) dots are data obtained under the (open) periodic boundary condition for the $y$ direction. For the $x$ direction, the periodic boundary condition is imposed. The number of sites for the $y$ direction is set to $L_{y}=20$.

a nontrivial value of the winding number $v_{ \pm}\left(k_{x}^{*}\right)$, nonHermiticity is essential.

The mirror winding number taking a nontrivial value results in a different skin effect, which is a main result of this Rapid Communication; in spite of $v_{\text {tot }}=0$, the energy eigenvalues significantly depend on the boundary condition for the mirror invariant line in the Brillouin zone. This skin effect should generically occur when the mirror winding number is finite because each subsector of the Hamiltonian corresponds to the one belonging to class $\mathrm{A}$, which shows the ordinary skin effect characterized by the winding number $\nu_{ \pm}$. Here, we stress that the mirror symmetry protects the topology inducing the skin effect [74,75].

In the following, we verify that the nontrivial topology characterized with a mirror winding number results in the above significant dependence by numerically analyzing a tight-binding model. The Hamiltonian reads

$$
\begin{aligned}
H(\boldsymbol{k})= & {\left[2 t\left(\cos k_{x}+\cos k_{y}\right)-\mu\right] \rho_{0}+i \Delta \sin k_{x} \rho_{3} } \\
& +i \Delta \sin k_{y} \rho_{2},
\end{aligned}
$$

where $\rho_{i}(i=1,2,3)$ are the Pauli matrices and $\rho_{0}$ is the $2 \times 2$ identity matrix. As discussed in Sec. I of the Supplemental Material [76], this model is related to a Hermitian system [77]. Furthermore, as discussed below, a system having the same topological properties of the above model can be realized for an electric circuit. The above non-Hermitian Hamiltonian preserves the mirror symmetry with $M_{x}=\rho_{2} P_{x}$. Therefore, for $k_{x}^{*}=0$ or $\pi$, the Hamiltonian can be block-diagonalized with $\rho_{2}$. For $k_{x}^{*}=0\left(k_{x}^{*}=\pi\right)$, the mirror winding number takes $v_{\mathrm{M}}=1$ with $E_{\mathrm{pg}}=2 t-\mu\left(E_{\mathrm{pg}}=-2 t-\mu\right)$, while the total winding number is zero for an arbitrary value of $k_{x}$.

In Fig. 2 the energy spectrum of the Hamiltonian (4) is plotted for $(t, \mu, \Delta)=(1,2,1.8)$ at $k_{x}=0, \pi / 6, \pi / 2, \pi$. The data denoted with blue (orange) dots represent the energy eigenvalues for the $\mathrm{PBC}(\mathrm{OBC})$ along the $y$ direction, respectively. Figure 2(a) indicates that the energy spectrum under the $\mathrm{PBC}$ forms a circle enclosing the origin of the complex 

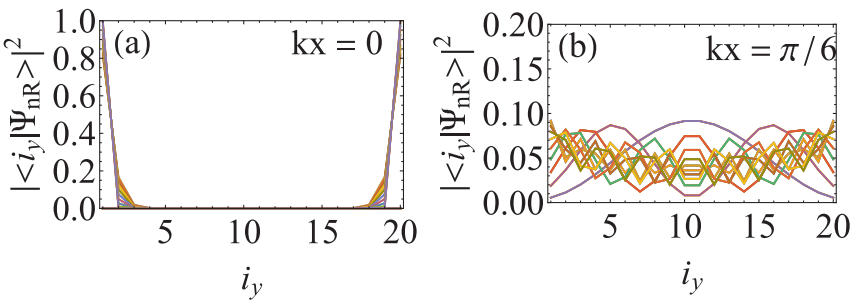

FIG. 3. (a) [(b)] Amplitude of the right eigenvectors of the Hamiltonian (4) for $(t, \mu, \Delta)=(1,2,1.8)$ at $k_{x}=0(\pi / 6)$, respectively. The data are obtained under the periodic (open) boundary condition for the $x(y)$ direction, respectively. The number of the sites along the $y$ direction is set to $L_{y}=20$.

plane, which is consistent with the relation $v_{\mathrm{M}}=1$ for $k_{x}=0$. Imposing the $\mathrm{OBC}$ along the $y$ direction significantly changes the spectrum; energy eigenvalues are aligned along the real axis (i.e., $\operatorname{Im} E_{n} \sim 0$ with $n=1,2, \ldots, \operatorname{dim} H$ ). This striking dependence of the energy spectrum is a signal of the skin effects. Here, we note that the mirror symmetry plays an essential role; away from the mirror invariant line, the spectra obtained for the two distinct boundary conditions coincide with each other [see Figs. 2(b) and 2(c)]. At $k_{x}=\pi$, the mirror symmetry is preserved, which again induces the skin effect [see Fig. 2(d)].

In association with the significant change in the energy eigenvalues, the eigenvectors show extensive localization. Figure 3 plots the amplitude of the right eigenvectors $\left|\left\langle i_{y} \mid \Psi_{n R}\right\rangle\right|^{2}$ for $k_{x}=0, \pi / 6$. Here, $\left|\Psi_{n R}\right\rangle$ denotes the right eigenvector of the Hamiltonian (4) (i.e., $H\left|\Psi_{n R}\right\rangle=\left|\Psi_{n R}\right\rangle E_{n}$ with $n=1, \ldots, \operatorname{dim} H$ ), and $i_{y}$ labels the sites along the $y$ direction. We note that the eigenstates are extended in the bulk under the PBC along the $y$ direction (see Sec. II A in the Supplemental Material [76]). Imposing the OBC for the $y$ direction results in an extensive localization of the eigenstates. Figure 3(a) shows that at the mirror invariant line $k_{x}=0$, all of the eigenstates for the Hamiltonian $H_{+}\left(k_{x}=0\right)\left[H_{-}\left(k_{x}=0\right)\right]$ are localized around $i_{y}=0\left(i_{y}=L_{y}\right)$, respectively, while the states are extended under the PBC. The anomalous localization under the $\mathrm{OBC}$ is another characteristic feature of the skin effect. We note that away from the mirror invariant line, the states localized around each edge are mixed and extend to the bulk [see Fig. 3(b)], which also indicates that the mirror symmetry is essential.

Based on the above significant difference of the energy spectra and the eigenstates, we can conclude that a nontrivial value of the mirror winding number $v_{M}$ results in the mirror skin effect. We note that adding a mirror-symmetry breaking term extinguishes the skin effect, which explicitly demonstrates that mirror symmetry protects the skin effect. For further detailed numerical analyses, see Secs. II and III of the Supplemental Material [76]. We furthermore propose that this mirror skin effect can be observed for an electric circuit composed of negative impedance converters (see Fig. 1), the details of which are discussed in the rest of this Rapid Communication.

Mirror skin effect in an electric circuit. Before giving a detailed proposal for the implementation of the circuit showing the mirror skin effect, let us briefly review how an electric circuit mimics a generic tight-binding Hamiltonian. Consider an electric circuit where the voltage $V_{a}(\omega)$ is applied at nodes $a=1,2, \ldots$ with angular frequency $\omega$. In this case, based on the Kirchhoff's law, the current $I_{b}(\omega)$ at node $b$ is given by

$$
I_{b}(\omega)=\sum_{a} J_{b a}(\omega) V_{a}(\omega) .
$$

Thus, the admittance matrix $J_{b a}(\omega)$ serves as a Hamiltonian for the corresponding tight-binding model, which means that topological phenomena can also be observed for electric circuits [13]. For instance, the Su-Schrieffer-Heeger model can be realized for an electric circuit composed only with capacitors and inductors. The energy conservation of the electric circuit implies the Hermiticity of the matrix $J_{b a}(\omega)$ up to the global phase factor $i$.

Now, let us discuss how to experimentally verify the mirror skin effect for electric circuits. In order to implement a circuit showing the mirror skin effect, we need to reproduce nonHermitian terms of the Hamiltonian [i.e., the second and the third term of Eq. (4)], which can be accomplished by employing the negative impedance converters $[78,79]$. Specifically, we propose that an electric circuit shown in Fig. 1 serves as a platform of the mirror skin effect. The corresponding admittance matrix is given by

$$
\begin{aligned}
\left(\begin{array}{l}
I_{A}(\omega, \boldsymbol{k}) \\
I_{B}(\omega, \boldsymbol{k})
\end{array}\right)= & J(\omega, \boldsymbol{k})\left(\begin{array}{l}
V_{A}(\omega, \boldsymbol{k}) \\
V_{B}(\omega, \boldsymbol{k})
\end{array}\right), \\
J(\omega, \boldsymbol{k})= & i \omega\left[-2 C_{0}\left(\cos k_{x}+\cos k_{y}\right) \rho_{0}\right. \\
& +\left(4 C_{0}+\frac{1}{\omega^{2} L_{0}}\right) \rho_{0} \\
& \left.+2 i C_{1} \sin k_{x} \rho_{3}+2 i C_{1} \sin k_{y} \rho_{1}\right],
\end{aligned}
$$

in the momentum space. $I_{\alpha}(\omega, \boldsymbol{k})$ and $V_{\alpha}(\omega, \boldsymbol{k})(\alpha=A, B)$ denote the Fourier transformed current and voltage, respectively [80,81]. The detailed derivation of Eq. (6) is given in Sec. IV A of the Supplemental Material [76]. This model preserves the mirror symmetry with $M_{x}=\rho_{1} P_{x}$. In addition, its topology is characterized by the mirror winding number taking $v_{\mathrm{M}}\left(k_{x}^{*}\right)=-1\left(k_{x}^{*}=0, \pi\right)$ for the parameter set summarized in the caption of Fig. 4. We note that the relation $v_{\text {tot }}=0$ holds for arbitrary momentum $k_{x}$. We mention here that the circuit elements of the above parameters are commercially available. Numerical data elucidating the above topological properties are shown in Sec. IV B of the Supplemental Material [76].

In the following, we see that the above model shows the mirror skin effect. For the electric circuit, the skin effect can be experimentally observed by measuring the admittance eigenvalues $j_{n}$ with $n=1, \ldots, \operatorname{dim} J$ [i.e., eigenvalues of the admittance matrix (6b)]. One can access the admittance eigenvalues by the impedance measurement $\left[J_{a b}^{-1}(\omega)\right][79]$. When the skin effect occurs, the admittance spectrum significantly depends on the boundary condition as we have seen in Fig. 2(a). Figure 4(a) [Fig. 4(b)] shows the admittance spectrum for $k_{x}=0\left(k_{x}=\pi / 6\right)$, respectively. For the momentum invariant line $k_{x}=0$, the admittance spectrum significantly changes depending on the boundary condition [see Fig. 4(a)]; the eigenvalues form a circle for the PBC, while they form 

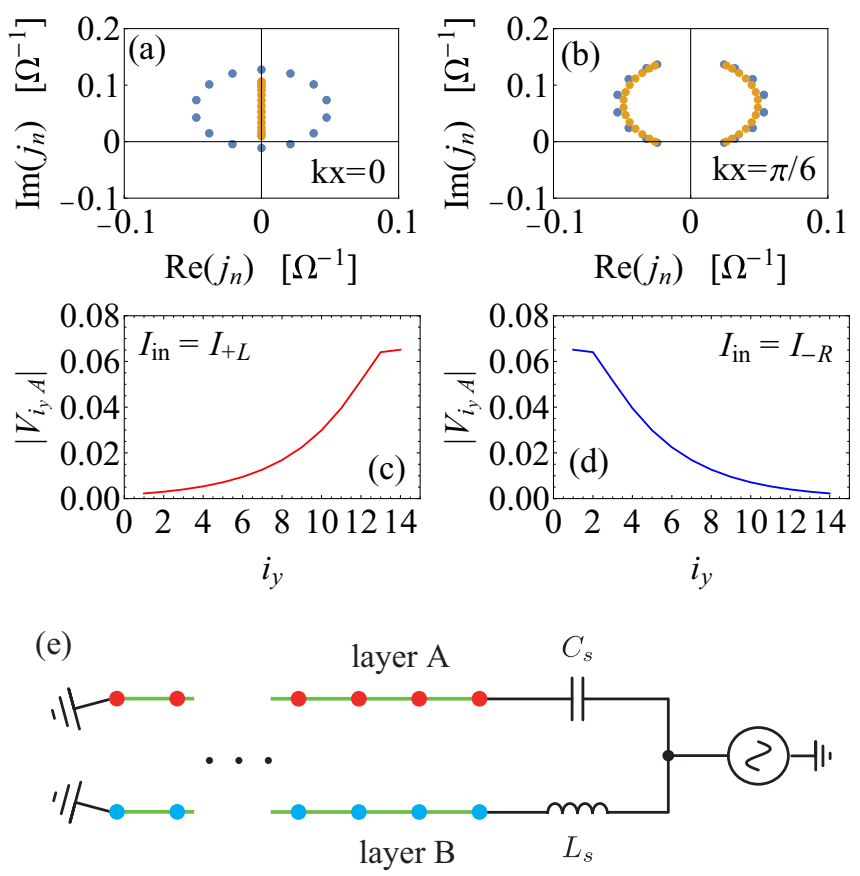

FIG. 4. (a) [(b)] Admittance spectrum for each case of the boundary condition for $k_{x}=0\left(k_{x}=\pi / 6\right)$, respectively. Blue (orange) dots denote the admittance eigenvalue $j_{n}$ under the periodic (open) boundary condition for the $y$ direction with $L_{y}=14$. The periodic boundary condition is imposed for the $x$ direction. The data are obtained for $L_{0}=120 \mu \mathrm{H}, C_{0}=47 \mathrm{nF}, C_{1}=33 \mathrm{nF}$, and $f=117.4 \mathrm{kHz}$, where $f$ denotes frequency $\omega=2 \pi f$. (c) [(d)] Voltage profile of layer A for the case where the current with the plus (minus) sector of reflection is fed. (e) Illustration of setup to observe the anomalous voltage response with $I_{\text {in }}=I_{+\mathrm{L}}$. For the above parameter sets, the fed current is $I_{\mathrm{s}}=0.0001$ A with a fed voltage $V_{\mathrm{s}}=0.002 \mathrm{~V}$ for $C_{\mathrm{s}}=0.068 \mu \mathrm{F}$, and $L_{\mathrm{s}}=27 \mu \mathrm{H}$.

a straight line for the OBC. Away from the mirror invariant line, the mirror operation is not closed for each momentum $k_{x}$, which results in the absence of the skin effect [see Fig. 4(b)].

Extensive localized states, observed in Fig. 3(a), are also observed through the voltage profile with the current feed for each mirror sector. In Fig. 4(c) [Fig. 4(d)], we plot the voltage profile with the current feed $I_{\text {in }}=I_{+L} \quad\left(I_{\text {in }}=\right.$ $\left.I_{-R}\right)$, respectively. Here, $I_{+L i_{y} \alpha}=i I_{s} \delta_{i_{y} L_{y}}\left(\delta_{\alpha A}+i \delta_{\alpha B}\right), I_{-\mathrm{L} i_{y} \alpha}=$ $i I_{s} \delta_{i_{0} 0}\left(\delta_{\alpha A}-i \delta_{\alpha B}\right)$, and $I_{s}=0.0001 \mathrm{~A}$. Tuning the phase of the feed current is accomplished with the setup illustrated in Fig. 4(e). In Fig. 4(c), we observe that the voltage response becomes large around the right edge $i_{y}=L_{y}$ although the current is fed at the left edge $i_{y}=0$. The essentially same result can also be observed for the other mirror sector [see Fig. 4(d)].
The above anomalous response for the feed current arises from the extensively localized states. First, we note that the voltage profile for inputted current can be obtained as $V_{a}=$ $\sum_{b} J_{a b}^{-1} I_{\text {in } b}$ with the impedance matrix $J_{a b}^{-1}=\sum_{n} R_{a n} j_{n}^{-1} L_{n b}^{\dagger}$ $[79,82]$. Here, the matrix $R\left(L^{\dagger}\right)$ denotes the set of the right (left) eigenvectors $\sum_{b} J_{a b} R_{b n}=R_{a n} j_{n}\left(\sum_{a} L_{n a}^{\dagger} J_{a b}=j_{n} L_{n b}^{\dagger}\right)$, respectively. $a$ and $b$ denote the set of the labels $i_{y}$ and $\alpha=A, B$. We note that $\sum_{n} L_{n b}^{\dagger} I_{+\mathrm{L} b}=0$ when $n$ labels the states for the minus sector. Thus, we can see that the only eigenvectors for the plus sector contribute to the voltage response. In addition, when one of the states $\left(n=n_{0}\right)$ for the plus sector is dominant [83], the voltage is estimated as $\left|V_{i_{y} A}\right| \sim\left|R_{i_{y} A n_{0}} j_{n_{0}}^{-1} \sum_{b} L_{n_{0} b}^{\dagger} I_{\text {in } b}\right|$. Therefore, we observe that the extensive localized states induce the anomalous voltage response for $I_{\text {in }}=I_{+L}$. The anomalous response shown in Fig. 4(d) can be understood in a similar way.

The above results indicate that the mirror skin effect can be observed for the circuit shown in Fig. 1. Specifically, by measuring the elements of the admittance matrix in the real space and by applying the Fourier transformation, one can experimentally observe the significant change of admittance eigenvalues for $k_{x}=0, \pi$ [Figs. 4(a) and 4(b)] [79,82]. In a similar way, one can experimentally observe the anomalous voltage response [Figs. 4(c) and 4(d)] by extracting the contribution of $k_{x}=0, \pi$ form data measured in the real space. We note that it can be another approach to measure the above quantities for the system where only a few sites are aligned along the $x$ direction.

Summary. In this Rapid Communication, we have analyzed the interplay between mirror symmetry and skin effects, shedding light on crystalline symmetry and non-Hermitian topology. Our analysis has clarified a type of skin effect, a mirror skin effect, which results in the significant dependence both of the energy spectrum and the states on the boundary condition only along mirror invariant lines in the two-dimensional Brillouin zone. The topological characterization of this skin effect can be done with the mirror winding number. The mirror skin effect has been verified by numerically diagonalizing a tight-binding Hamiltonian with the mirror winding number taking one. Here, we stress that the discovery of the skin effect protected by mirror symmetry is a key result rather than introducing the mirror winding number.

Furthermore, we have proposed how to implement the electric circuit for the experimental observation of the mirror skin effect. In this system, switching the boundary condition significantly changes the admittance eigenvalues, which serves as distinct evidence of the mirror skin effect.

Acknowledgments. This work is partly supported by JSPS KAKENHI Grants No. JP16K13845, No. JP17H06138, No. JP18H05842, and No. JP20H04627. Parts of the numerical calculations were performed on the supercomputer at the ISSP in the University of Tokyo.
[1] M. Z. Hasan and C. L. Kane, Rev. Mod. Phys. 82, 3045 (2010).

[2] X.-L. Qi and S.-C. Zhang, Rev. Mod. Phys. 83, 1057 (2011).

[3] Y. Hatsugai, Phys. Rev. Lett. 71, 3697 (1993).
[4] F. D. M. Haldane and S. Raghu, Phys. Rev. Lett. 100, 013904 (2008).

[5] S. Raghu and F. D. M. Haldane, Phys. Rev. A 78, 033834 (2008). 
[6] Z. Wang, Y. Chong, J. D. Joannopoulos, and M. Soljacic, Nature (London) 461, 772 (2009).

[7] C. L. Kane and T. C. Lubensky, Nat. Phys. 10, 39 (2013).

[8] T. Kariyado and Y. Hatsugai, Sci. Rep. 5, 18107 (2015).

[9] R. Süsstrunk and S. D. Huber, Science 349, 47 (2015).

[10] S. D. Huber, Nat. Phys. 12, 621 (2016).

[11] P. Delplace, J. B. Marston, and A. Venaille, Science 358, 1075 (2017).

[12] V. V. Albert, L. I. Glazman, and L. Jiang, Phys. Rev. Lett. 114, 173902 (2015).

[13] C. H. Lee, S. Imhof, C. Berger, F. Bayer, J. Brehm, L. W. Molenkamp, T. Kiessling, and R. Thomale, Commun. Phys. 1, 39 (2018).

[14] J. C. Y. Teo, L. Fu, and C. L. Kane, Phys. Rev. B 78, 045426 (2008).

[15] L. Fu, Phys. Rev. Lett. 106, 106802 (2011).

[16] T. H. Hsieh, H. Lin, J. Liu, W. Duan, A. Bansil, and L. Fu, Nat. Commun. 3, 982 (2012).

[17] Y. Tanaka, Z. Ren, T. Sato, K. Nakayama, S. Souma, T. Takahashi, K. Segawa, and Y. Ando, Nat. Phys. 8, 800 (2012).

[18] K. Hashimoto, X. Wu, and T. Kimura, Phys. Rev. B 95, 165443 (2017).

[19] W. A. Benalcazar, B. A. Bernevig, and T. L. Hughes, Science 357, 61 (2017).

[20] F. Schindler, A. M. Cook, M. G. Vergniory, Z. Wang, S. S. P. Parkin, B. A. Bernevig, and T. Neupert, Sci. Adv. 4, eaat0346 (2018).

[21] W. A. Benalcazar, B. A. Bernevig, and T. L. Hughes, Phys. Rev. B 96, 245115 (2017).

[22] S. Hayashi, Commun. Math. Phys. 364, 343 (2018).

[23] S. Imhof, C. Berger, F. Bayer, J. Brehm, L. W. Molenkamp, T. Kiessling, F. Schindler, C. H. Lee, M. Greiter, T. Neupert, and R. Thomale, Nat. Phys. 14, 925 (2018).

[24] H. Araki, T. Mizoguchi, and Y. Hatsugai, Phys. Rev. B 99, 085406 (2019).

[25] S. A. A. Ghorashi, X. Hu, T. L. Hughes, and E. Rossi, Phys. Rev. B 100, 020509(R) (2019).

[26] K. Kudo, T. Yoshida, and Y. Hatsugai, Phys. Rev. Lett. 123, 196402 (2019).

[27] T. Mizoguchi, M. Maruyama, S. Okada, and Y. Hatsugai, Phys. Rev. Mater. 3, 114201 (2019).

[28] N. Hatano and D. R. Nelson, Phys. Rev. Lett. 77, 570 (1996).

[29] Y. C. Hu and T. L. Hughes, Phys. Rev. B 84, 153101 (2011).

[30] K. Esaki, M. Sato, K. Hasebe, and M. Kohmoto, Phys. Rev. B 84, 205128 (2011).

[31] A. Guo, G. J. Salamo, D. Duchesne, R. Morandotti, M. Volatier-Ravat, V. Aimez, G. A. Siviloglou, and D. N. Christodoulides, Phys. Rev. Lett. 103, 093902 (2009).

[32] C. E. Rüter, K. G. Makris, R. El-Ganainy, D. N. Christodoulides, M. Segev, and D. Kip, Nat. Phys. 6, 192 (2010).

[33] A. Regensburger, C. Bersch, M.-A. Miri, G. Onishchukov, D. N. Christodoulides, and U. Peschel, Nature (London) 488, 167 (2012).

[34] B. Zhen, C. W. Hsu, Y. Igarashi, L. Lu, I. Kaminer, A. Pick, S.-L. Chua, J. D. Joannopoulos, and M. Soljacic, Nature (London) 525, 354 (2015).

[35] A. U. Hassan, B. Zhen, M. Soljačić, M. Khajavikhan, and D. N. Christodoulides, Phys. Rev. Lett. 118, 093002 (2017).

[36] T. E. Lee, Phys. Rev. Lett. 116, 133903 (2016).
[37] Y. Xu, S.-T. Wang, and L.-M. Duan, Phys. Rev. Lett. 118, 045701 (2017).

[38] T. Yoshida, K. Kudo, and Y. Hatsugai, Sci. Rep. 9, 16895 (2019).

[39] V. Kozii and L. Fu, arXiv:1708.05841..

[40] T. Yoshida, R. Peters, and N. Kawakami, Phys. Rev. B 98, 035141 (2018).

[41] K. Kimura, T. Yoshida, and N. Kawakami, Phys. Rev. B 100 , 115124 (2019).

[42] A. A. Zyuzin and A. Y. Zyuzin, Phys. Rev. B 97, 041203(R) (2018).

[43] M. Papaj, H. Isobe, and L. Fu, Phys. Rev. B 99, 201107(R) (2019).

[44] T. Matsushita, Y. Nagai, and S. Fujimoto, Phys. Rev. B 100, 245205 (2019).

[45] T. Yoshida, R. Peters, N. Kawakami, and Y. Hatsugai, arXiv:2002.11265.

[46] K. Kawabata, K. Shiozaki, M. Ueda, and M. Sato, Phys. Rev. X 9, 041015 (2019).

[47] H. Shen, B. Zhen, and L. Fu, Phys. Rev. Lett. 120, 146402 (2018).

[48] K. Kawabata, S. Higashikawa, Z. Gong, Y. Ashida, and M. Ueda, Nat. Commun. 10, 297 (2019).

[49] Z. Gong, Y. Ashida, K. Kawabata, K. Takasan, S. Higashikawa, and M. Ueda, Phys. Rev. X 8, 031079 (2018).

[50] H. Zhou and J. Y. Lee, Phys. Rev. B 99, 235112 (2019).

[51] J. C. Budich, J. Carlström, F. K. Kunst, and E. J. Bergholtz, Phys. Rev. B 99, 041406(R) (2019).

[52] R. Okugawa and T. Yokoyama, Phys. Rev. B 99, 041202(R) (2019).

[53] H. Zhou, J. Y. Lee, S. Liu, and B. Zhen, Optica 6, 190 (2019).

[54] T. Yoshida, R. Peters, N. Kawakami, and Y. Hatsugai, Phys. Rev. B 99, 121101(R) (2019).

[55] K. Kawabata, T. Bessho, and M. Sato, Phys. Rev. Lett. 123, 066405 (2019).

[56] T. Yoshida and Y. Hatsugai, Phys. Rev. B 100, 054109 (2019).

[57] J. Carlström and E. J. Bergholtz, Phys. Rev. A 98, 042114 (2018).

[58] T. Katō, Perturbation Theory for Linear Operators (Springer, Berlin, 1966), Vol. 132.

[59] I. Rotter, J. Phys. A: Math. Theor. 42, 153001 (2009).

[60] V. M. Martinez Alvarez, J. E. Barrios Vargas, and L. E. F. Foa Torres, Phys. Rev. B 97, 121401(R) (2018).

[61] F. K. Kunst, E. Edvardsson, J. C. Budich, and E. J. Bergholtz, Phys. Rev. Lett. 121, 026808 (2018).

[62] S. Yao and Z. Wang, Phys. Rev. Lett. 121, 086803 (2018).

[63] S. Yao, F. Song, and Z. Wang, Phys. Rev. Lett. 121, 136802 (2018).

[64] E. Edvardsson, F. K. Kunst, and E. J. Bergholtz, Phys. Rev. B 99, 081302(R) (2019).

[65] W. B. Rui, Y. X. Zhao, and A. P. Schnyder, Phys. Rev. B 99, 241110(R) (2019).

[66] K. Yokomizo and S. Murakami, Phys. Rev. Lett. 123, 066404 (2019).

[67] N. Okuma and M. Sato, Phys. Rev. Lett. 123, 097701 (2019).

[68] L. Xiao, T. Deng, K. Wang, G. Zhu, Z. Wang, W. Yi, and P. Xue, Nat. Phys. (2020), doi:10.1038/s41567-020-0836-6.

[69] C. H. Lee and R. Thomale, Phys. Rev. B 99, 201103(R) (2019).

[70] K. Zhang, Z. Yang, and C. Fang, arXiv:1910.01131. 
[71] N. Okuma, K. Kawabata, K. Shiozaki, and M. Sato, Phys. Rev. Lett. 124, 086801 (2020).

[72] C.-H. Liu, H. Jiang, and S. Chen, Phys. Rev. B 99, 125103 (2019).

[73] Topological classification with mirror symmetry can be carried out for the bulk [72]. For two-dimensional systems, the classification result of symmetry class $\mathrm{A}$ is $\mathbb{Z}$. This topological properties are characterized by the mirror winding number. The key result of this Rapid Communication is elucidating that the bulk topology results in the skin effect protected by mirror symmetry.

[74] W. B. Rui, M. M. Hirschmann, and A. P. Schnyder, Phys. Rev. B 100, 245116 (2019).

[75] The mirror skin effect discussed here differs from the one mentioned in Ref. [74] where applying the mirror operator exchanges the left and right edges. Furthermore, our work elucidates the relation between the skin effect and the point-gap topology with mirror symmetry, which is a key result of our work .
[76] See Supplemental Material at http://link.aps.org/supplemental/ 10.1103/PhysRevResearch.2.022062 for a relation between a Hermitian system, details of numerical results, and details of the electric circuit .

[77] H. Yao and S. Ryu, Phys. Rev. B 88, 064507 (2013).

[78] T. Hofmann, T. Helbig, C. H. Lee, M. Greiter, and R. Thomale, Phys. Rev. Lett. 122, 247702 (2019).

[79] T. Helbig, T. Hofmann, S. Imhof, M. Abdelghany, T. Kiessling, L. W. Molenkamp, C. H. Lee, A. Szameit, M. Greiter, and R. Thomale, Nat. Phys. (2020).

[80] We note that employing diodes [81] is considered to be another approach to realize the skin effect .

[81] M. Ezawa, Phys. Rev. B 99, 201411(R) (2019).

[82] T. Hofmann, T. Helbig, F. Schindler, N. Salgo, M. Brzezińska, M. Greiter, T. Kiessling, D. Wolf, A. Vollhardt, A. Kabaši et al., Phys. Rev. Research 2, 023265 (2020).

[83] We note that inductors $L_{0}$ are essential for making one of the eigenvalues $j_{n_{0}}^{-1}$ become dominant. 